\title{
Tener hermanos mayores y concurrir a guarderías en edades tempranas se asociaron a un menor riesgo de asma en edades mayores
}

Sibling, day-care attendance, and the risk of asthma and wheezing during childhood. Ball TM, Castro-Rodriguez JA, Griffith KA, et al. N Engl J Med 2000; 343: 538-43.

\section{Objetivo}

Determinar en niños pequeños si la presencia de hermanos mayores en el hogar y la asistencia temprana a guarderías disminuye la frecuencia de episodios de sibilancias y disminuye el riesgo de padecer asma en edades mayores.

\section{Diseño}

Estudio de cohorte prospectivo*

\section{Lugar}

Muestra poblacional de Tucson, Arizona, EE.UU.

\section{Participantes}

Cohorte de 1035 niños, seguidos desde el nacimiento hasta los 13 años.

\section{Evaluación de factores pronósticos}

Cuestionario contestado por los padres relativo al estado respiratorio de sus hijos a los $6,8,11$ y 13 años del estudio. Se relevaba número y edad de los hermanos. Se definió asistencia temprana a la guardería (presencia de por lo menos 6 niños) cuando la concurrencia fue antes de los 6 meses de vida. A los pacientes en seguimiento a los 6 y 11 años de edad, se les realizó tests de sensibilidad cutáneas a alergenos comunes del área y dosaje séricos de $\lg \mathrm{E}$.

\section{Medición del resultado principal}

Se consideró como episodios sibilantes frecuentes cuando presentaban más de 3 episodios sibilantes en el último año. Se consideró asma a por lo menos 1 episodio diagnosticado como tal por un profesional cuando el paciente tenía entre 6 a 13 años.

\section{Resultados principales}

La presencia de uno o más hermanos en el hogar resultó un factor protector para el desarrollo subsecuente de asma (RR ajustado por cada hermano adicional: 0.8, IC95\% 0.7 a 1.0, p=0.04), así como también la concurrencia temprana a guarderías durante los seis primeros meses de vida (RR ajustado 0.4, IC95\% 0.2 a 1.0 , $\mathrm{p}=0.04)$. Se observó que los niños con mayor exposición con otros niños en casas o guarderías presentaron mayor frecuencia de episodios sibilantes antes de cumplir los 2 años de vida en comparación con aquellos con escasa o nula exposición temprana con otros niños (RR ajustado 1.4, IC95\% 1.1 a 1.8, p=0.01), pero presentaron menor número de episodios sibilantes desde los 6 a 13 años de vida.

\section{Conclusiones}

La exposición de niños pequeños con sus hermanos mayores en el hogar o con otros niños en guarderías, son factores protectores para el desarrollo de asma y de episodios sibilantes frecuentes en la infancia tardía.

Fuente de financiamiento: National Heart, Lung, and Blood Institute.

\section{Comentario}

El asma bronquial es la enfermedad crónica más común en la infancia, con una prevalencia aproximada del 10 al 15\%. Es una de las principales causas de ausentismo escolar y de indicaciones de restricción de la actividad física. A pesar de la dificultad de estandarizar los criterios diagnósticos para determinar la morbilidad exacta de ésta patología, se ha observado un aumento alarmante de su incidencia y prevalencia en los últimos 20 años, motivando una serie de trabajos para explicar éste fenómeno. ${ }^{1}$ Uno, encontró que muchos lactantes tienen cuadros de sibilancias en los primeros meses de vida asociados especialmente a episodios infecciosos virales, presentando además disminución de la función pulmonar al nacimiento que no incrementan el riesgo de asma o alergia más tarde en la vida. Se encontró que las sibilancias en los primeros 3 años de vida tuvieron buen pronóstico. A pesar de que $1 / 3$ de los menores de 3 años presentan sibilancias, cerca del $60 \%$ de éstos niños dejan de presentarlas a los 6 años. Por otro lado aquellos mayores de 6 años con sibilancias persistentes presentaron niveles sanguíneos de Ig E elevados a los 9 meses de vida. ${ }^{2}$

Este trabajo tiene la importancia de realizar un seguimiento prolongado de un número importante de lactantes. Tiene utilidad para determinar las probables causas del aumento importante de la incidencia de asma infantil, sustentando la hipótesis de que el menor número de infecciones respiratorias en lactantes en los primeros meses de vida podría ser la causa de éste aumento de la incidencia, sobre todo en las poblaciones urbanas. Es en estas donde el número de hijos por pareja está en franco descenso, sien- do los hermanos mayores los principales focos de contagio de cuadros respiratorios virales.

Se realizaron otros estudios en relación con el número de hermanos y la disminución de la frecuencia de diversos desordenes alérgicos en edades mayores, pero cabe destacar que los periodos de seguimiento no fueron muy prolongados. ${ }^{3}$

Si bien están claramente establecidos los factores predisponentes del asma como: antecedentes familiares de asma, prolongación de la lactancia materna exclusiva hasta los 6 meses de edad 4 y e tabaquismo materno durante el periodo perinatal, ha sido más difícil establecer cuáles son los factores protectores. Este trabajo demuestra claramente la relación directa de cuadros sibilantes a repetición en los primeros años de vida, en niños con ingreso precoz a guarderías y con mayor número de hermanos convivientes: relación que se torna protectora para las sibilancias y el asma a partir de los 6 años de vida.

Los problemas encontrados en el estudio son: la frecuencia de aparición de crisis de asma y sibilancias está basada en respues. tas de los cuestionarios hechos a los padres, que si bien aprenden a reconocer las crisis luego de varios episodios, no todos ellos fueron constatados por profesionales para determinar con efectividad la presencia de sibilancias, y los datos del inicio de la guardería fueron tomados retrospectivamente varios años después. Si bien los resultados del trabajo no generan impacto con respecto a las normas de diagnóstico y tratamiento, explicaría las alteraciones actuales del patrón epidemiológico, desviando el foco de las causas exógenas como principal explicación de éste patrón.

Dr. Pascual Barán [. Unidad de Medicina Familiar y Preventiva. Hospital Italiano de Buenos Aires ]

\section{Referencias}

Worldwide variations in the prevalence ofmasthma syntoms: the International Study of Asthma and Allegies in Childhood (ISA AC). Eur Respir J 1998; 12: 315-335.

2. Martinez F, Wright AL, Taussing LM y col. Asthma and wheezing in the first six years of life. N Eng J Med 1995; 332: 133-138.

3. Ponsonby AL Couper D. Dwyer T Carmichael A. Cross sectional study of the relation between sibling number and asthma, hay fever, and eczema. Arch Dis Child 1998; 79: 328-333. 4 Wilson A, Stewart Forsyth J, Greenes, et al. Relation of infant diet to childhood health: seven year folow up of cohort of children in Dundee infant feeding study. BMJ 1998; 316: 21-25. 